\title{
2-Dimensional Chromatography
}

National Cancer Institute

\section{Source}

National Cancer Institute. 2-Dimensional Chromatography. NCI Thesaurus. Code C161768.

The use of two chromatography-based techniques in sequence to separate the various substances in a mixture. 